\title{
Distribution and quantitative variation of mitochondrial plasmid-like DNAs in cultivated rice (Oryza sativa $\mathbf{L}$.)
}

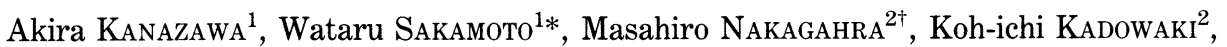 \\ Nobuhiro TsuTsumi ${ }^{1}$ and Shigemitsu TANO ${ }^{1}$ \\ ${ }^{1}$ Laboratory of Radiation Genetics and Chemical Mutagenesis, Faculty of \\ Agriculture, The University of Tokyo, 1-1-1 Yayoi, Bunkyo-ku, Tokyo 113 \\ ${ }^{2}$ National Institute of Agrobiological Resources, 2-1-2 \\ Kannondai, Tsukuba, Ibaraki 305, Japan
}

(Received 16 March 1992)

\begin{abstract}
Distribution of four kinds of mitochondrial plasmid-like DNAs, B1, B2, B3 and B4 was investigated by Southern analysis using 85 accessions of cultivated rice (Oryza sativa L.). The frequencies of these molecules found in cultivars differed from each other. The mitochondrial genome were categorized into nine types according to their presence or absence. They were found mostly in ecospecies Indica, a few in Javanica, and not found in Japonica. The result indicated the polymorphic and monomorphic patterns of the mitochondrial genomic organization within Indica and Japonica cultivars, respectively. Quantitative variation was found among four kinds of mitochondiral plasmid-like DNAs, suggesting that these molecules were unequally distributed or replicated during the process of mitochondrial division.
\end{abstract}

\section{INTRODUCTION}

Higher plant mitochondria often contain extrachromosomal small circular and linear DNA molecules (Pring and Lonsdale, 1985). These DNAs are called plasmid-like DNAs. Four kinds of rice mitochondrial plasmid-like DNAs have been detected and analyzed in DNA sequence (Yamaguchi and Kakiuchi, 1983; Shikanai et al., 1987, 1989; Shikanai and Yamada, 1988). Kadowaki et al. (1988b) examined the diversity of mitochondrial plasmid-like DNAs by gel electrophoresis and classified the cytoplasms into three types based on the electrophoretic mobilities of the molecules. Homologous nucleotide sequences with these molecules were detected in the nuclear genome. It was suggested recently that these DNA sequences were transferred via RNA (Fukuchi et al., 1991). The hybridization pattern of these nuclear homologues showed restriction fragment polymorphisms (RFLPs) among varietal groups of O. sativa and between O. sativa

* Present address: Boyce Thompson Institute for Plant Research at Cornell University, Tower Road, Ithaca, New York, 14853, USA

+ Present address: Secretariat for Agriculture, Forestry and Fisheries Research Council, Ministry of Agriculture, Forestry and Fisheries, Kasumigaseki, 1-2-1, Chiyoda-ku, Tokyo 100, Japan 
and O. glaberrima. A genetical study revealed that the nuclear homologues were located on chromosomes and inherited consistently during the varietal differentiation of cultivated rice (Sakamoto et al., 1991). Some of the nuclear homologues linked tightly with each other, and the linkage was conserved among several rice cultivars (Kanazawa et al., 1991). Thus the mitochondrial plasmid-like DNAs and the nuclear homologues of these molecules were proposed to be useful as genetic markers for these two genomes. In this paper, we examined the identity and the distribution of the plasmid-like DNAs, B1, B2, B3 and B4, among cultivated rice (O. sativa L.) by Southern analysis. Based on the data, we investigated the relationship of the type of mitochondrial genome to the varietal groups of cultivated rice so far classified and to the geographical distribution of the materials. We also examined the relative copy number of these molecules in several rice cultivars as an approach to elucidate the diversity of their existence in mitochondria.

\section{MATERIALS AND METHODS}

\section{Plant Materials}

Eighty-five accessions of rice cultivars provided by National Institute of Agrobiological Resources, Japan, were used to investigate the distribution of mitochondrial plasmid-like DNAs. These cultivars were either indigenous or pure lines, consisted of 35 Indica cultivars, 17 Sinica (Chinese hsien type) cultivars, 18 Javanica cultivars and 15 Japonica cultivars according to the classification of Nakagahra (1978). Four cultivars were used for the quantitative assay of plasmid-like DNAs. Cultivars Chinsurah boro II, IR 24 and Jamuna were Indica, and Cms-Bo Taichung No. 65 was Japonica but carrying the cytoplasm of Chinsurah boro II.

\section{DNA preparation}

Mitochondrial DNAs were extracted from etiolated leaves according to the methods of Kadowaki et al. (1988a). Plasmid-like DNAs (B1, B2, B3 and B4) cloned previously (Sakamoto et al., 1989; Kanazawa et al., 1991) were used as probes for hybridization.

\section{Southern hybridization and quantitative analysis of mt plasmid-like DNAs}

For the examination of the distribution of plasmid-like DNAs, mitochondrial DNAs (mtDNAs) from each of the cultivars were separated electrophoretically in $0.7 \%$ agarose gels, blotted to nitrocellulose filters and hybridized with the cloned B1, B2, B3 and B4 DNAs.

To compare the relative copy number among plasmid-like DNAs, mtDNAs were separated in $1 \%$ agarose gel after the triple digestion with BglII, EcoRV and $M l u \mathrm{I}$, by which each plasmid-like DNA was converged to single linear molecule. 
The blotted filters were hybridized with mixed probes of B1, B2 and B4, or B1, B3 and B4, independently. The B2 and B3 DNAs were not used simultaneously due to their close migration in the gel. Probe DNAs were recovered from the recombinant plasmid and labelled with $\left[\alpha^{32} \mathrm{P}\right] \mathrm{dCTP}$ using the multiprime DNA labelling kit (Amersham, $3000 \mathrm{Ci} / \mathrm{mmol}$ ). Pre-hybridization was carried out in $6 \times \mathrm{SSPE}, 50 \%$ formamide, $5 \times$ Denhardt's solution, $0.5 \%$ SDS, and $100 \mu \mathrm{g} / \mathrm{ml}$ salmon sperm DNA at $42^{\circ} \mathrm{C}$ over night. Hybridization was done in $6 \times \mathrm{SSPE}$, $50 \%$ formamide, $0.5 \%$ SDS, $100 \mu \mathrm{g} / \mathrm{ml}$ salmon sperm DNA, and $25 \mathrm{ng} /$ filter labelled DNA at $42^{\circ} \mathrm{C}$ over night. The filters were washed twice in $2 \times \mathrm{SSC}, 0.1 \%$ $\mathrm{SDS}$ at $42^{\circ} \mathrm{C}$ for $10 \mathrm{~min}$, twice in $1 \times \mathrm{SSC}, 0.1 \% \mathrm{SDS}$ at $42^{\circ} \mathrm{C}$ for $10 \mathrm{~min}$, and in $0.1 \times \mathrm{SSC}, 0.1 \% \mathrm{SDS}$ at $65^{\circ} \mathrm{C}$ for $30 \mathrm{~min}$. The washed filters were exposed to $\mathrm{X}$-ray film with an intensifying screen.

For the quantitative assay, the corresponding bands were cut out from the filters and the radioactivity of the hybridized probes was measured in the liquid scintillation counter. The uniformity of the transfer efficiency of DNAs and the saturation of the hybrid formation between the probe and its target DNAs were checked by the hybridization in which the amount of DNAs applied in the gel was varied.

\section{RESULTS}

\section{Differential distribution of plasmid-like DNAs observed in O. sativa}

The mitochondrial plasmid-like DNAs B1, B2, B3 and B4 were identified in 9, 46, 44 and 37 cultivars, respectively, by Southern analysis. The cultivars examined here were classified into nine types with regard to the presence or absence of these molecules in mitochondria (Table 1). The B1 DNA was found at comparatively low frequency. The other notable finding was the co-existence of different kinds of plasmid-like DNAs found frequently for B2 and B3. These two

Table 1. Mitochondrial genome types based on the presence or absence of mitochondrial plasmid-like DNAs

\begin{tabular}{|c|c|c|c|c|c|c|c|c|c|c|}
\hline \multirow{2}{*}{$\begin{array}{l}\text { Varietal } \\
\text { group }\end{array}$} & \multicolumn{9}{|c|}{ Mitochondrial genome type } & \multirow{2}{*}{ Tota } \\
\hline & b0 & $\mathrm{b} 2$ & $\mathrm{~b} 4$ & $\mathrm{~b} 23$ & $\mathrm{~b} 24$ & b123 & b134 & b234 & b1234 & \\
\hline Indica & 2 & 0 & 1 & 1 & 2 & 1 & 1 & 21 & 6 & 35 \\
\hline Sinica & 3 & 0 & 0 & 8 & 0 & 1 & 0 & 5 & 0 & 17 \\
\hline Javanica & 16 & 1 & 1 & 0 & 0 & 0 & 0 & 0 & 0 & 18 \\
\hline Japonica & 15 & 0 & 0 & 0 & 0 & 0 & 0 & 0 & 0 & 15 \\
\hline Total & 36 & 1 & 2 & 9 & 2 & 2 & 1 & 26 & 6 & 85 \\
\hline
\end{tabular}

The numbers in the names of mitochondrial genome type indicate the plasmid-like DNAs presented in mitochondria (e.g.: b0, no plasmid-like DNA was identified; b2, B2 was identified but B1, B3 and B4 were not; b1234, B1, B2, B3 and B4 were identified). 
plasmid-like DNAs were identified together in 43 cultivars (93\% and $98 \%$ of the cultivars having $\mathrm{B} 2$ and $\mathrm{B} 3$, respectively). These results suggest that each plasmid-like DNA was unequally transmitted during the varietal differentiation in rice cultivars.

Table 2. Presence or absence of mitochondrial plasmid-like DNAs in Indica rice cultivars

\begin{tabular}{llllll}
\hline \hline Cultivar & Origin & B1 & B2 & B3 & B4 \\
\hline Jaguary & Brazil & - & - & - & - \\
Amyong & India & - & - & - & + \\
Anjana & India & - & + & + & + \\
Ausjhoria & India & - & + & + & - \\
Auslaljira & India & - & + & + & + \\
Auspaddy & India & - & + & + & + \\
Black gora & India & + & - & + & + \\
Buramada & India & - & + & + & + \\
Chakhodarongbi & India & - & + & - & + \\
Deola & India & - & + & - & + \\
DR 18687 & India & + & + & + & + \\
IRI 183 & India & + & + & + & + \\
Jhangi 34 & India & + & + & + & + \\
Kainon & India & - & + & + & + \\
Kalamanik & India & + & + & + & + \\
Kelam & India & + & + & + & + \\
Kolu & India & - & + & + & + \\
N 22 & India & - & + & + & + \\
Nabai dhan & India & - & + & + & + \\
Ramajowan & India & - & + & + & + \\
Rella wadlu & India & - & + & + & + \\
Sadatusuku & India & + & + & + & + \\
Sathi & India & - & + & + & + \\
Sonajuti & India & - & + & + & + \\
Sonamukhi & India & - & + & + & + \\
Surjamkhi & India & - & + & + & + \\
Thurunga & India & - & - & - & - \\
Trinaikuppy & India & - & + & + & + \\
Tong-I & Korea & - & + & + & + \\
Jena 015 & Nepal & - & + & + & + \\
Nepal No. 18 & Nepal & + & + & + & - \\
IR 29 & Philippine & - & + & + & + \\
IR 2061-214-3 & Philippine & - & + & + & + \\
Kinandang puti & Philippine & - & + & + & + \\
Dakanalo & & - & + & + & + \\
\hline & & & & & +
\end{tabular}


Table 3. Presence or absence of mitochondrial plasmid-like DNAs in Sinica rice cultivars

\begin{tabular}{llllll}
\hline \hline Cultivar & Origin & B1 & B2 & B3 & B4 \\
\hline Daizau & North China & - & + & + & - \\
Aijiau nan teh & South China & - & + & + & + \\
Dau zen chiau & South China & - & + & + & - \\
Guangru ai No. 4 & South China & - & - & - & - \\
Guechan No. 2 & South China & - & + & + & + \\
Hai & South China & - & - & - & - \\
Hong mi & South China & - & + & + & + \\
Liuzou bauya zau & South China & - & + & + & - \\
Shuen chung mi & South China & - & + & + & - \\
Siligu & South China & - & + & + & - \\
Simong huang pigu & South China & + & + & + & - \\
Taichung No. 3 & South China & - & + & + & + \\
Taichung yu No. 204 & South China & - & + & + & + \\
Zai yeh chin No. 8 & South China & - & - & - & - \\
Chinyu & Taiwan & - & + & + & - \\
Duanguang hua lei & Taiwan & - & + & + & - \\
Wagu hua lei & Taiwan & - & + & + & - \\
\hline
\end{tabular}

Table 4. Presence or absence of mitochondrial plasmid-like DNAs in Javanica rice cultivars

\begin{tabular}{llllll}
\hline \hline Cultivar & Origin & B1 & B2 & B3 & B4 \\
\hline Dourado precoce & Brazil & - & - & - & + \\
In sitt & Myanmar & - & - & - & - \\
Khauk yoe & Myanmar & - & - & - & - \\
Masumikir & Indonesia & - & - & - & - \\
Padi kenikir putih & Indonesia & - & - & - & - \\
Siampang & Indonesia & - & - & - & - \\
Dam ngo & Laos & - & - & - & - \\
Mong wang gu & North China & - & - & - & - \\
Cana bong bong & Philippine & - & + & - & - \\
Cs-s 4 & Philippine & - & - & - & - \\
Dinalaga & Philippine & - & - & - & - \\
Dabai gu & South China & - & - & - & - \\
Gang & South China & - & - & - & - \\
Monang gu & South China & - & - & - & - \\
Sen nong 1033 & South China & - & - & - & - \\
North rose & USA & - & - & - & - \\
Texas fortuna & USA & - & - & - & - \\
Vista & USA & - & - & - & - \\
\hline
\end{tabular}


Table 5. Precence or absence of mitochondrial plasmid-like DNAs in Japonica rice cultivars

\begin{tabular}{llllll}
\hline \hline Cultivar & Origin & B1 & B2 & B3 & B4 \\
\hline Romeo & Italy & - & - & - & - \\
Shinriki & Japan & - & - & - & - \\
Beiji & North China & - & - & - & - \\
Hung gu & North China & - & - & - & - \\
Dali 635-781 & South China & - & - & - & - \\
Jinning 26-11-4 & South China & - & - & - & - \\
Lizu hong & South China & - & - & - & - \\
Simong gu & South China & - & - & - & - \\
Yun 21 & South China & - & - & - & - \\
Yun 83-128 & South China & - & - & - & - \\
Yun 83-132 & South China & - & - & - & - \\
Yun 83-133 & South China & - & - & - & - \\
Yun 83-141 & South China & - & - & - & - \\
Yun 83-142 & South China & - & - & - & - \\
Yun 83-147 & South China & - & - & - & - \\
\hline
\end{tabular}

\section{Relationship with varietal groups}

The relationship between the distribution of these molecules and the varietal groups of the rice cultivars were investigated. The cultivars subjected with the experiment were categorized into Indica, Sinica, Javanica and Japonica, according to the classification based on the isozymic and phylogenetic studies of Nakagahra (1978). The presence or absence of the mitochondrial plasmid-like DNAs in each cultivar was shown in Tables 2-5. The pattern was highly polymorphic in Indica and Sinica varieties. In contrast, no plasmid-like DNA was identified in Japonica varieties. In Javanica varieties, plasmid-like DNAs were found at low frequency (in two cultivars out of 18) (Table 1). The frequencies of the cultivars which lacked all of the four molecules in mitochondria were 0.06 (two cultivars out of 35), 0.18 (three out of 17) and 0.89 (16 out of 18), in Indica, Sinica and Javanica, respectively. The mean numbers of the kinds of plasmid-like DNAs found in one cultivar were calculated by dividing the sum of the number of the kinds of plasmid-like DNAs in each cultivar by the number of cultivars. Those of Indica, Sinica and Javanica were 2.86, 2.00 and 0.11 , respectively.

\section{Geographical distribution of mitochondrial plasmid-like DNAs}

Geographical distribution of the plasmid-like DNAs was examined according to the origin of the cultivars used. Plasmid-like DNAs were identified in much of the cultivars from India and its neighboring districts such as Nepal and Sri Lanka. Twenty-eight cultivars out of 30 from these districts had at least one plasmid-like DNA, among which large diversity was observed. The mean number of the 
kinds of plasmid-like DNAs found in one cultivar was 2.90 in these cultivars. In the cultivars from Southeast Asia, from South China and Taiwan, and from North China and Korea, the numbers were 1.08, 1.03 and 1.00, respectively. Six types of mitochondrial genome regarding the presence or absence of these molecules were observed in the cultivars from India (b0, b23, b24, b134, b234 and b1234). Four types: b0, b23, b123 and b234, and three types: b0, b2 and b234 were observed in the cultivars from South China and Southeast Asia, respectively.

\section{Quantitative variation among plasmid-like DNAs}

Differential distribution of each of the four plasmid-like DNAs were found among rice cultivars as described above. In the next place, we investigated whether the relative copy numbers of each plasmid-like DNA were conserved among cultivars. MtDNAs were digested to linearize each plasmid-like DNA, in order to converge various hybridized signals of multimers (Fig. 1) to single signal for exact quantitation. After the treatment, DNAs were separated by gel electrophoresis, transferred to filters and hybridized with mixtures of labelled plasmid-like DNAs (Fig. 2). The relative copy number of plasmid-like DNAs were estimated by the hybridized amount of probe DNAs after correction for size

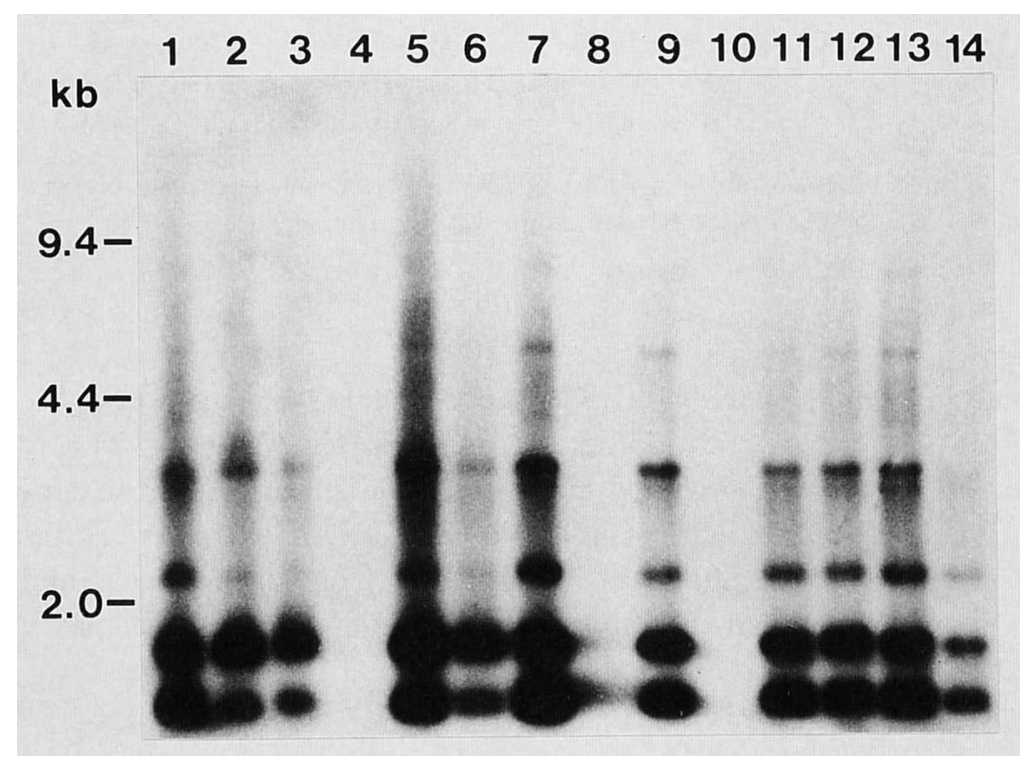

Fig. 1. Hybridization of the mitochondrial plasmid-like DNA B3 to the mtDNAs from various rice cutivars. MtDNAs from each cultivars were electrophoresed, transferred to a filter and hybridized with labelled B3. Lane 1, Sathi (Indica); lane 2, Jhangi 34 (Indica); lane 3, IRI 183 (Indica); lane 4, Thurunga (Indica); lane 5, Kelam (Indica); lane 6, IRI 183 (Indica); lane 7, Hong mi (Sinica); lane 8, Dourado precoce (Javanica); lane 9, Taichung No. 3 (Sinica); lane 10, Hung gu (Japonica); lane 11, Kinandang puti (Indica); lane 12, Duanguang hua lei (Sinica); lane 13, Guechan No. 2 (Sinica); lane 14, Wagu hua lei (Sinica). 


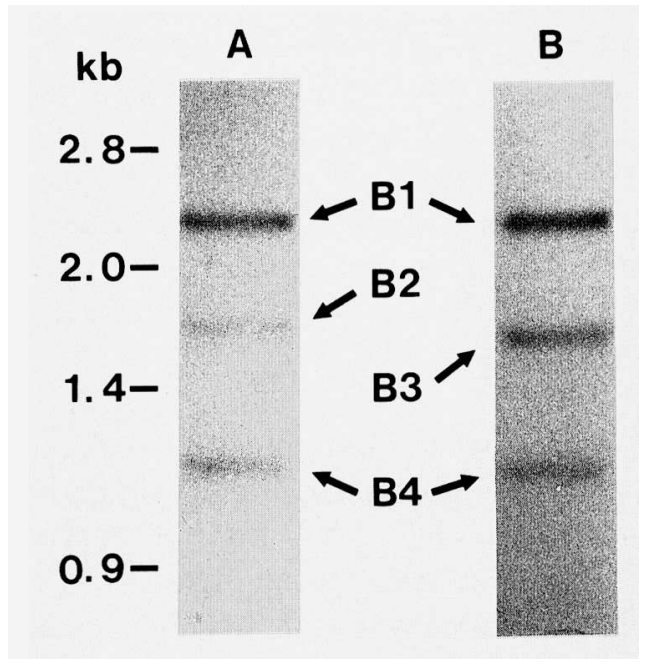

Fig. 2. Hybridization for the estimation of relative copy number of mitochondrial plasmidlike DNAs in Chinsurah boro II. MtDNAs were digested with $B g l \mathrm{II}, E c o$ RI and $M l u \mathrm{I}$ to linearize plasmid-like DNAs, separated by electrophoresis and transferred to filters. The filters were probed with the mixtures of labelled B1, B2 and B4 DNAs (A), and B1, B3 and B4 DNAs (B).

Table 6. Relative copy number of mitochondrial plasmid-like DNAs

\begin{tabular}{lcccc}
\hline \hline Cultivar & B1 & B2 & B2 & B4 \\
\hline Chinsurah boro II & $0.831 \pm 0.090^{\mathrm{a}}$ & $0.497 \pm 0.043$ & $0.845 \pm 0.065$ & $1^{\mathrm{b}}$ \\
Cms-Bo Taichung No. 65 & $0.802 \pm 0.095$ & $0.480 \pm 0.005$ & $0.833 \pm 0.028$ & 1 \\
IR 24 & $-^{\mathrm{c}}$ & $0.495 \pm 0.003$ & $1.193 \pm 0.007$ & 1 \\
Jamuna & $0.489 \pm 0.016$ & $0.834 \pm 0.028$ & $1.474 \pm 0.049$ & 1 \\
\hline
\end{tabular}

a The mean value \pm standard deviation calculated by two or more independent experiments

b The amount of hybridized probe B4 was assigned a copy number of 1 .

c_, Not found

differences.

The results of quantitative assay for four cultivars, Chisurah boro II, Cms-Bo Taichung No. 65, IR 24 and Jamuna were shown in Table 6. The hybridized amount for B4 was assigned a copy number of 1 . Difference of the copy number was found among four kinds of plasmid-like DNAs in every cultivar. The relativity in their copy number was conserved in Chinsurah boro II and Cms-Bo Taichung No. 65, which had the same cytoplasm and different nucleus, whereas it was conserved neither in IR 24 nor in Jamuna.

\section{DISCUSSION}

The composition of mitochondrial genome showed much diversity among rice cultivars. Nine types were observed regarding the presence or absence of the four kinds of mitochondrial plasmid-like DNAs. These molecules were not found at the same frequency. B1 DNA was rarely identified: it was found in the ratio from one fourth to one fifth of others. B1 and B3 DNAs always existed with 
other kind of plasmid-like DNAs. We found the tendency of B2 and B3 DNAs to co-exist except in only three cultivars. These results suggested that the distribution of plasmid-like DNAs was biased during the varietal differentiation of cultivated rice.

Kadowaki et al. (1988b) classified rice cultivars into three groups according to the electrophoretic patterns of mitochondrial plasmid-like DNAs. Our results of Southern analysis probed with mitochondrial plasmid-like DNAs essentially confirmed the report. However, more diversity was found in this experiment.

Classification of cultivated rice $(O$. sativa) has been approached from various viewpoints. Based on the degree of hybrid seed fertility, Morinaga (1954) and Chang (1976) proposed Javanica as an intermediate ecotype between two wellknown subspecies, Indica and Japonica. Nakagahra $(1977,1978)$ separated Sinica group from Indica group according to the isozymic and phylogenetic analyses. These traits depend on the nuclear genome. Our present results, using cytoplasmic trait, indicate that the presence or absence of the plasmid-like DNAs, B1, B2, B3 and B4 in mitochondria is generally consistent with such classifications. These plasmid-like DNAs were found mostly in the cultivars of ecospecies Indica, whereas they were not found in ecosp. Japonica. There were, however, some cultivars which lacked plasmid-like DNAs among ecosp. Indica. Within the cultivars so far investigated, more diversity was observed in Indica than in Sinica. The polymorphic pattern, and monomorphic or at most only a few patterns of the genomic organization have been reported for Indica and Japonica, respectively, for chloroplast genome (Ishii et al. 1988; Dally and Second, 1990), and for nuclear genome (Second, 1982; Glaszmann, 1987; Cordesse et al., 1990; Takaiwa and Oono, 1991). Our data indicates that these findings are applicable to mitochondrial genome.

Plasmid-like DNAs, as well as their diversity, were identified much in the cultivars from India and its neighboring districts. Nakagahra (1978) reported the genetic diversity tended to be simpler in the areas remote from the candidate districts for the origin of $O$. sativa by isozymic analysis. In our present study, two general tendencies were observed. One was that the diversity of the composition of mitochondrial genome was much in the cultivars from the districts ranging from India to South China. The other was that the observed frequency of the plasmid-like DNAs became lower in the districts far away from there.

We found the quantitative variation among the plasmid-like DNAs in the cells of leaves in several cultivars. There were differences in the relative copy number among four kinds of plasmid-like molecules. The relativity was conserved between Chinsurah boro II and Cms-Bo Taichung 65, which had the same cytoplasm, but not conserved in IR 24 and Jamuna, implying that it was typical of each cytoplasm. The variation suggests unequal distribution of plasmid-like DNA molecules during the mitochondrial division, or replication of each molecules in different efficiency in mitochondria. Such unequal transmission may probably 
contribute to the diversity of the presence or absence of mitochondrial plasmidlike DNAs among rice cultivars.

We wish to express our appreciation to Dr. Tatara, The University of Tokyo, for his valuable suggestions.

\section{REFERENCES}

Chang, T. T. (1976). The origin, evolution, cultivation, dissemination, and diversification of Asian and African rices. Euphytica 25, 425-441.

Cordesse, F., Second, G. and Deleseny, Y. (1990). Ribosomal gene spaser length variability in cultivated and wild rice species. Theor. Appl. Genet. 79, 81-88.

Dally, A. M. and Second, G. (1990). Chloroplast DNA diversity in wild and cultivated species of rice (Genus Oryza, section Oryza). Cladistic-mutation and genetic-distance analysis. Theor. Appl. Genet. 80, 209-222.

Fukuchi, M., Shikanai, T., Kossykh, V. G. and Yamada, Y. (1991). Analysis of nuclear sequences homologous to the B4 plasmid-like DNA of rice mitochondria; evidence for sequence transfer from mitochondria to nuclei. Curr. Genet. 20, 487-494.

Glaszmann, J. C. (1987). Isozymes and classification of Asian rice varieties. Theor. Appl. Genet. 74, 21-30.

Ishii, T., Terachi, T. and Tsunewaki, K. (1988). Restriction endonuclease analysis of chloroplast DNA from A-genome diploid species of rice. Jpn. J. Genet. 63, 523-536.

Kadowaki, K., Osumi, T., Nemoto, H., Harada, K. and Shinjyo, C. (1988a). Mitochondrial DNA polymorphism in male-sterile cytoplasm of rice. Theor. Appl. Genet. 75, 234-236.

Kadowaki, K., Yazaki, K., Osumi, T., Harada, K., Katsuta, M. and Nakagahra, M. (1988b). Distribution of mitochondrial plasmid-like DNA in cultivated rice (Oryza sativa L.) and its relationship with varietal groups. Theor. Appl. Genet. 76, 809-814.

Kanazawa, A., Sakamoto, W., Kishimoto, N., Yano, M., Tsutsumi, N., Saito, A. and Tano, S. (1991). Linkage analysis of the nuclear homologues of mitochondrial plasmid-like DNAs in rice. Jpn. J. Genet. 66, 597-607.

Morinaga, T. (1954). Classification of rice varieties on the basis of affinity. Jpn. J. Breed. 4, 1-14.

Nakagahra, M. (1977). Genic analysis for esterase isoenzymes in rice cultivars. Jpn. J. Breed. 27, 141-148.

Nakagahra, M. (1978). The differentiation, classification and genetic diversity of cultivated rice (Oryza sativa L.) by isozyme analysis. Trop. Agric. Res. 11, 77-82.

Pring, D. R. and Lonsdale, D. M. (1985). Molecular biology of higher plant mitochondrial DNA. Int. Rev. Cytol. 97, 1-46.

Sakamoto, W., Momose, M., Tsutsumi, N., Tano, S. and Yamaguchi, H. (1989). Analysis of homology of small plasmid-like mitochondrial DNAs in the different cytoplasmic male sterile strains in rice. Jpn. J. Genet. 64, 49-56.

Sakamoto, W., Kadowaki, K., Kishimoto, N., Yano, M., Saito, A. and Tano, S. (1991). RFLP analysis of nuclear DNAs homologous with mitochondrial plasmid-like DNAs in cultivated rice. Theor. Appl. Genet. 82, 179-184.

Second, G. (1982). Origin of the genetic diversity of cultivated rice (Oryza spp.): study of the polymorphism scored at 40 isozyme loci. Jpn. J. Genet. 57, 25-57.

Shikanai, T., Yang, Z. Q. and Yamada, Y. (1987). Properties of the circular plasmid-like DNA B1 from mitochondria of cytoplasmic male-sterile rice. Plant Cell Physiol. 28, 1243-1251.

Shikanai, T. and Yamada, Y. (1988). Properties of the circular plasmid-like DNA, B4, from mitochondria of cytoplasmic male-sterile rice. Curr. Genet. 13, 441-443. 
Shikanai, T., Yang, Z. Q. and Yamada, Y. (1989). Nucleotide sequence and molecular characterization of plasmid-like DNAs from mitochondria of cytoplasmic male-sterile rice. Curr. Genet. 15, 349-354.

Takaiwa, F. and Oono, K. (1991). Restriction fragment length polymorphism of glutelin from cultivated rice (Oryza sativa L.). Jpn. J. Genet. 66, 155-160.

Yamaguchi, H. and Kakiuchi, H. (1983). Electrophoretic analysis of mitochondrial DNA from normal and male sterile cytoplasms in rice. Jpn. J. Genet. 58, 607-611. 